\title{
7 Validación de CoMpetencias EN Francia
}

Tomás Díaz González

Universidad de Valladolid

\section{INTRODUCCIÓN}

El proceso de "Validación de Competencias en Francia" tiene 15 años de historia. El Decreto 85/906, del 23 de agosto, pone en marcha un proceso que no hace sino ampliarse día a día extendiendo e introduciéndose en el Sistema Educativo y Laboral. Esta experiencia francesa ha recorrido en el tiempo procesos idénticos a la experiencia de Validación en el Reino Unido. Las filosofías y métodos no son coincidentes debido a los modelos de sistemas sociales de ambos países.

La Unión Europea a través de los programas LEONARDO DA VINCI Y SÓCRATES esta impulsando experiencias en este terreno.

Los nuevos aires de movilidad social de los trabajadores y la necesidad de una Europa unida que busca un sistema de certificación y de libre circulación hace que la certificación sea una herramienta apropiada.

El trabajo que presento se basa en dos fuentes:

- Mi contacto con grandes actores de Validación en Francia, gracias a un permiso de movilidad del profesorado que me fue concedido por el Ministerio de Educación y Cultura; y 
- Gracias a la participación en el proyecto titulado "EUROVALIDACION DE COMPETENCIAS", junto con las universidades y empresas de Francia y Reino Unido financiado por el programa LEONARDO.

Parto de un marco general de Certificación en Europa para situar la validación en Francia, donde sitúo sobre todo el marco legislativo. Dentro del conjunto de modelos que describo, quiero explicitar tres líneas aplicativas a la realidad española donde la legislación es muy distinta:

- Modelo de Colegios Cooperativos, vinculados a ciertas universidades.

- Metodologías del "portafolio" que sigue una línea más escolar; y

- Validación en empresas, fundamentalmente en el sector minerometalúrgico.

Acompaño bibliografía resumida de la amplia producción que sobre el tema se ha realizado en los 15 años de experimentación; igualmente he resumido un conjunto de siglas habituales en Francia para el tema de Validación.

\section{SISTEMA GENERAL DE CERTIFICACIÓN EN EUROPA}

Teniendo en consideración lo dictaminado por CEDEFOP (1992), cuando nos referimos al término "certificación" aludimos con ello al hecho de expedir un diploma, certificado u otro título con el aval de las autoridades, organismos oficiales de formación y personas competentes para tal fin. Tomando en consideración lo mencionado con anterioridad, pasaremos a presentar un resumen del análisis comparativo de los sistemas y procedimientos de certificación existentes en la actualidad en el ámbito de acción francés.

\subsection{CREACIÓN Y APLICACIÓN DE LOS TÍTULOS DE FORMACIÓN PROFESIONAL}

\subsubsection{Responsabilidades legales}

En la mayoría de los Estados Miembros de la Comunidad Europea, la responsabilidad última de la atribución de los títulos destinados a certificar un determinado nivel de cualificación recae sobre el Ministerio de Educación. También puede suceder que esta función la desempeñe el Ministerio de Trabajo, como ocurre con todos los aspectos relacionados con la Formación Profesional Continua, aunque siempre será el Ministerio de Educación Nacional el organismo oficial en el cual recaerá la autoridad que garantice al título su carácter oficial y, con ello, su validez y homologación en todo el territorio nacional. 
A pesar de lo descrito hasta ahora, debemos reconocer que existe en todos los lugares un gran número de títulos concedidos al margen de los procedimientos nacionales oficiales de certificación. Un ejemplo de todo ello es el ámbito de influencia de validación de competencias que no es otro que el de la Formación Continua, donde dichos certificados sólo tienen un valor en función del renombre del organismo que lo expide. Para paliar este efecto varios países han implantado un sistema denominado de homologación.

\subsubsection{Papel desempeñado por los Interlocutores o Agentes Sociales}

En el caso de Francia, los diferentes sectores profesionales pueden determinar y desarrollar diversos procesos de formación que desemboquen en cualificaciones reconocidas por los Convenios Colectivos. A su vez, en cada una de las actividades profesionales que componen estos sectores pueden producirse solicitudes de homologación de determinadas competencias desarrolladas en el ejercicio de las tareas profesionales que componen cada actividad. Los Interlocutores Sociales están presentes en las Comisiones Paritarias Consultivas cuyo trabajo consiste en elaborar o actualizar los títulos de Formación Profesional. La decisión de homologación incumbe en todo caso al Ministerio Titular de Trabajo por delegación del primer Ministro.

\section{HOMOLOGACIÓN DE COMPETENCIAS EN FRANCIA}

\subsection{SENTIDO DE LA HOMOLOGACIÓN}

La complejidad del sistema de promoción profesional francés hace que la validación y el reconocimiento de competencias pongan en tela de juicio toda una terminología sabiendo que el vocabulario no se ha estabilizado, sino que progresivamente se ha llegado a un consenso de la literatura especializada. Tres términos aparecen claros: certificación, reconocimiento y validación. Seguimos los trabajos de A. M. Charraud (1994) representante del
CEREQ. La certificación se entiende de manera general como la operación que permite garantizar, autentificar y legalizar las competencias y el saber hacer del individuo.

Esta definición implica: por una parte, a actores que han recibido una responsabilidad legal y que se comprometen en el tema; y por otra parte, señala la relatividad de este compromiso con respecto al campo de competencias del actor o de la institución que certifica. 
En Francia hace referencia a una norma formalizada "oficial" que garantiza que quien es portador de un certificado domina los conocimientos, el saber hacer y los programas de formación que están reflejados en el diploma. Hay tres campos: los diplomas, los títulos homologados y los certificados de cualificación profesional.

\section{A. Diplomas}

El diploma es el documento más antiguo. Es un monopolio del Estado conferido esencialmente al Ministerio de Educación Nacional. El diploma hace referencia a una norma oficial, ya sea un referencial de diploma, ya sea una maqueta; dos principios caracterizan este campo: la afirmación del carácter nacional de los diplomas, controlados por el Estado y la definición de diplomas profesionales con consultas que reúnen actores de la formación.

\section{B. Títulos homologados}

La Ley de 1971 sobre la Enseñanza Tecnológica ha estipulado que los títulos y diplomas de la enseñanza Tecnológica se puedan adquirir por las vías escolares universitarias, por el aprendizaje y por la Formación Profesional Continua. La ley precisa que los títulos están inscritos en un catálogo o lista de homologación; se puede decir que esta Ley de 1971 abre nuevas formas de crear títulos y certificados a través de la fórmula de homologación realizada a través de la comisión de homologación que obtuvo una estructura más estable en el decreto Ley del primero de octubre de 1990, diario oficial del 3 de octubre del 90, pero que había tenido su origen en el Quinto Plan (1969). Como títulos homologados por derecho están ya todos los que forman parte del Ministerio de Educación Nacional. Para el resto de organismos que no forman parte de dicho Ministerio deben entrar en un procedimiento llamado de "homologación"; incluso las universidades pueden someter a dicha comisión de homologación los llamados "títulos propios de la universidad". La comisión antes aludida recibe el nombre de "Comisión Técnica de homologación de títulos y diplomas", puesta bajo la autoridad del Ministerio de Trabajo después de pasar una consulta de expertos en el marco del organismo que pide el título. Actualmente en torno a 3000 títulos o diplomas han pasado por este procedimiento; todos ellos están clasificados según los seis niveles adoptados en el quinto Plan de 1969.

\section{Certificados de Cualificación Profesional (CQP)}

Estos certificados son fruto de la Ley de 1984 (Ley del 24 de Febrero del 84) que pone en marcha la obligación de negociar al nivel de ramas profesionales los objetivos y los medios de la Formación Profesional; estos certifica- 
dos son títulos creados y entregados en el marco de una rama profesional a través de una instancia paritaria donde participan organizaciones patronales y sindicales en el marco de acuerdos colectivos que organizan el proceso de la creación y la puesta en marcha.

Estos certificados tienen carácter operativo, se sitúan en la lógica de las competencias, tienden a realizarse por vía de alternancia y generalmente tratan de certificar aquellas profesiones nuevas que el mercado obliga a poner en marcha y que no han sido cubiertas por otros diplomas; este tipo de certificación se ofrece con frecuencia como contrato a los jóvenes en las empresas. Muchas veces estos contratos se presentan como alternativa a los diplomas del Ministerio de Educación Nacional. En este momento, en torno a 20 ramas profesionales han creado los certificados de cualificación profesional, pero el número es inferior al que ofrece el Ministerio de Educación Nacional. Por ello muchos responsables piden pasarelas para obtener diplomas nacionales.

Las ramas profesionales que más se han destacado son las industrias agroalimentarias, las industrias harineras, las carreteras y materiales, transporte de ruta, el comercio y la reparación del automóvil, las azucareras, destilerías y refinado de azúcar, pero especialmente ha sido notable la Unión de Industrias Minerometalurgias (U.I.M.M) cuyo método aparece en el punto 6 de este trabajo, así como los trabajos realizados en el marco de la Asociación Nacional de Formación del Automóvil (A.N.F.A.C). Podemos ver Anexo I, el proceso de elaboración de diplomas y títulos en Francia.

\subsection{PROCESO DE ELABORACIÓN DE TÍTULOS CERTIFICADOS}

Podemos distinguir las siguientes fases:

\section{a. Declaración de intenciones del solicitante:}

- Carta de declaración de intenciones del Organismo de Formación correspondiente.

- Contacto con el corresponsal de la Comisión Regional de Homologación.

- Puesta en marcha de un dossier tipo.

\section{b. Paso a la Comisión Técnica de Homologación}

- Transmisión del dossier acreditativo desde el Organismo de Formación a las autoridades, las cuales lo harán llegar a las siguientes estancias oficiales: 
- Gobernador Regional.

- Presidente del Consejo Regional.

- Director de Educación.

- Examen de las condiciones de homologación de la petición del título en el que tomarán como indicadores de dicho proceso los siguientes:

- Objetivos.

- Naturaleza profesional.

- Adecuación de la temporalización al proceso cualificador.

- Grado de solidez de la formación establecida.

- Clarificación reglamentaria del Organismo de Formación.

- Sesión extraordinaria de la Comisión Técnica de Homologación.

\section{c. Período de instrucción de la Comisión Técnica}

- Examen de la viabilidad del título:

- Objetivos de la formación y su adecuación a las necesidades del entorno.

- Adecuación a las condiciones de admisión establecidas.

- Designación de un portavoz por parte de la Comisión Técnica de Homologación.

- Determinación del cuerpo técnico.

\section{d. Decisión de la Comisión Técnica}

- Comunicación del dossier instruido a la Comisión Técnica de Homologación nacional.

- Decisión de homologación.

- Aparición en el Boletín Oficial. 


\section{MODELO FRANCÉS: DISPOSITIVO DE LA UNIVERSIDAD FRANCESA SOBRE VALIDACIÓN DE COMPETENCIAS PERSONALES Y PROFESIONALES ${ }^{\prime}$}

\subsection{ORIGEN DEL DISPOSITIVO. BREVE REFERENCIA HISTÓRICA}

Existe un consenso generalizado con respecto al origen de la puesta en marcha del Sistema de Validación de Competencias en Francia. Éste tiene su origen en diversas experiencias realizadas junto con Canadá en torno a los años 80. Por aquella época los Ministerios de Trabajo y Educación Nacional de Francia desarrollaron, juntamente con los de Canadá, un modelo que pudiera servir para la formación de jóvenes fracasados para quienes se busca un dispositivo de formación capaz de reinsertarles, nuevamente, en la sociedad. En una primera propuesta se pone en marcha lo que se denominó las metodologias portafolios o portafolios de competencias. Al extenderse la experiencia al ámbito de los adultos con la participación de la Universidad, se ponen en marcha diferentes reformas que darán lugar al Decreto 85/906 sobre las Competencias Personales y Profesionales que abarcarán a los diferentes niveles educativos existentes tras la conclusión de la etapa de Bachillerato.

\subsection{TEXTOS QUE DEFINEN LA VALIDACIÓN DE COMPETENCIAS PERSONALES Y PROFESIONALES EN FRANCIA}

A continuación, presentamos los textos que se encargan de regular y organizar la Validación de Competencias en las diferentes Etapas de Enseñanza Superior:

1) Decreto 85/906, de 23 de agosto, donde se fijan las condiciones de Validación de Estudios en vistas al acceso a la Enseñanza Superior.

2) Ley $92 / 678$, de 20 de Julio, relativa a la Validación de Competencias para la entrega de diplomas acreditativos.

3) Decreto 93/538, de 26 de Marzo, sobre la Validación de Competencias.

4) Decisión de 27 de Marzo de 1993 (Diario Oficial de 28-3-1993).

\footnotetext{
'En todo este apartado se utilizan materiales elaborados dentro del Provecto Eurovalidación llevado a cabo por diversas universidades de nacionalidad francesa, inglesa y española.
} 


\subsubsection{Objetivo de la Validación de Competencias}

El Decreto 85/906, mencionado anteriormente, permite entrar en distintos niveles que componen la Enseñanza Reglada tras la realización del Bachillerato, sin necesidad de poseer el diploma requerido para ello, de manera oficial. Es decir, es posible para el candidato ser dispensado de la acreditación académica correspondiente sobre la base de sus competencias profesionales en el área educativa a la que opta.

El Decreto 93/538 al que nos hemos referido permite reconocer la posesión, por parte del profesional, de un porcentaje diploma a través de la convalidación de las unidades de valor, módulos, créditos, etc. que lo componen, aunque no será posible obtener la convalidación total del diploma por este procedimiento.

\subsubsection{Campos de Aplicación}

El Decreto 85/906 permite validar todos los diplomas y títulos nacionales expedidos por Organismos pertenecientes al Ministerio de Enseñanza Superior. Pero el Decreto 93/538 excluye de este procedimiento a los estudios de medicina, odontológicos y farmacéuticos.

\subsubsection{Condiciones de Aceptación de las Demandas}

Para poder beneficiarse de lo dictaminado por el Decreto 85/906 es necesario haber interrumpido los estudios al menos dos años antes respecto del número de cursos que componen la titulación correspondiente, o tres menos cuando los ha interrumpido por fracaso escolar. En este caso, el aspirante deberá ser mayor de veinte años. Para beneficiarse del Decreto 93/538 el candidato debe haber ejercido una actividad profesional de manera continuada, durante al menos cinco años. Las prácticas integradas para la obtención de un diploma no se tendrán en cuenta.

\subsubsection{Procedimiento de Aceptación de la Candidatura}

Cada candidato debe rellenar un dossier que permitirá a las Instancias Universitarias facilitar la toma de decisiones. Según el Decreto 85/906 el candidato elaborará su propio dossier con las competencias implicadas en la formación de la acreditación a la que opta. La lista de documentos a entregar y las fechas son fijadas anualmente y, de manera particular, para cada proceso de validación y por cada Organismo de Formación. Este Organismo puede ser el encargado de la realización del dossier anteriormente mencionado. 
En el marco del Decreto de 93/538, el candidato no puede optar a más de un Certificado de Validación por año, estando obligado el Organismo Formador a publicar los plazos en los que se conseguirá el mencionado certificado.

\subsubsection{Instancias y Toma de Decisiones}

El Decreto 85/906 establece la existencia de una Comisión Pedagógica cuya composición está determinada por el Presidente de la Institución Universitaria o por el responsable del Organismo Formador. Éste fija el número y designa los miembros de la Comisión, definiendo su funcionamiento. Cada Comisión es presidida por un profesor y debe incluir, al menos, dos docentes del área de formación objeto de la validación, responsabilizándose uno de ellos de aquellas actividades de formación continua. Además, estas comisiones pueden incluir profesionales externos al Organismo correspondiente.

Por otro lado, se prevé la designación, por parte del Presidente de la Institución Universitaria o del responsable del Organismo, de Jurados de Validación encargados de examinar las solicitudes de validación.

\subsubsection{Contenido del Dossier}

La legislación que regula el desarrollo de la Enseñanza Superior hace alusión al conjunto de competencias tanto profesionales como sociales y personales. Esto es debido a que las formaciones de carácter superior intentan construir capacidades transversales, siendo estas capacidades las que se tratan de clarificar en el Dossier.

\subsection{MODOS DE APLICACIÓN DE LA LEGISLACIÓN EN MATERIA DE VALIDACIÓN}

\subsubsection{Definición del Procedimiento}

Las reglas de funcionamiento de los Organismos de Enseñanza Superior se caracterizaban por su autonomía y heterogeneidad, antes de 1994. En este marco, la función de la Administración Central era la de sensibilizar, incitar y promocionar las iniciativas al respecto. No podíamos encontrar entre los Organismos de Enseñanza Superior, y especialmente en la Universidad, un modelo único de aplicación de la legislación de validación de competencias profesionales. Desde el punto de vista práctico, la puesta en marcha de la aplicación de regulación legislativa sobre validación fue confiada, en un primer momento, a la Oficina de Formación Permanente de la Dirección General de Enseñanza Superior del Ministerio de Educación 
Nacional francés. Aunque dada la complejidad de esta misión, así como la relativa ruptura que imponían las diversas perspectivas sobre los procesos de validación con respecto a las prácticas clásicas, se impuso la necesidad de crear un nuevo grupo de trabajo que reuniera a responsables de RR.HH de empresas más significativas de cada sector correspondiente, junto con los actores del mundo universitario. Esta colaboración dio lugar al procedimiento que ha sido implantado en todos los Organismos Formadores por parte del Ministerio, a partir de Octubre de 1994. Esta metodología comprende cuatro etapas:

1. Entrevista al candidato por parte del asesor de formación para determinar la razón de la demanda.

2. Constitución del dossier bajo tutela del asesor.

3. Instrucción del dossier: El jurado aprecia las aportaciones del candidato.

4. Toma de decisiones del jurado, determinando los indicadores y elementos de control de conocimientos y competencias a lo largo del Proceso de Validación de las Competencias Profesionales.

\subsubsection{Seguimiento del Dispositivo}

En vista a sensibilizar a los Organismos Formadores en el proceso de Validación y con el objetivo de capitalizar las prácticas eficientes y ayudar a superar las resistencias, la Dirección General de Enseñanza Superior ha organizado, en dos ocasiones, en la Universidad de Lille I, diversas jornadas sobre validación de competencias profesionales entre los años 1993 y 1996. Estas jornadas, que han venido reuniendo en torno a trescientos universitarios, han dado lugar a la publicación de diferentes Actas ampliamente difundidas, contribuyendo a la integración progresiva de las diferentes prácticas existentes en la materia. En mayo de 1997, la Dirección General de Enseñanza Superior decidió lanzar un Plan de Acción Estratégico destinado a favorecer el desarrollo de la Validación de Competencias.

\subsection{RESULTADOS OBTENIDOS: MODELOS DE PUESTA EN MARCHA}

Hay tres modelos de puesta en marcha del Proceso de Validación de Competencias Profesionales en el seno de las universidades francesas. Un estudio reciente, dirigido por el Ministerio de Educación Nacional y realizado por la Universidad de Ciencias de Lille, ha demostrado que podemos identificar en estas universidades francesas hasta un total de tres modelos de puesta en marcha del mencionado Proceso de Validación. 


\section{Modelo: Una organización centralizada}

Se caracteriza por la creación de una Comisión Central o un Jurado Único, de geometría variable en su composición, que generalmente está presidido por el Vicepresidente de Estudios y de Escolaridad, donde está incluido el responsable de la Formación Continua del Organismo Formador que dirige el Proceso de Validación. Se puede decir que el Organismo de Formación Continua es el motor del proyecto.

\section{Modelo: Una organización descentralizada}

Se caracteriza por la existencia de dos propiedades:

a) Una organización en la que podríamos afirmar que su característica principal es la de la anomia, la desintegración de las normas que aseguraban un cierto orden y homogeneidad en el proyecto. Aquí, serán los docentes los que, de manera aislada, deciden por cuenta propia el modo y las unidades de valor que compondrán el proceso de validación. Cada profesor trabaja aisladamente y no hay una visión de conjunto.

b) Se produce una validación deslocalizada. Se trata de una práctica que se inscribe como prolongación de las prácticas de equivalencia aplicadas para la admisión en el proceso de formación inicial, existiendo comisiones propias en cada departamento.

\section{3. ${ }^{\text {er }}$ Modelo: Una organización administrativa}

Los Dossier de Validación son gestionados por los Servicios de Escolaridad o por el Servicio de Validación de Competencias, dirigido tanto a los estudiantes como a los adultos. El nivel de escolaridad alcanzado determina el dossier del participante, siendo éste transmitido a las comisiones puestas en marcha por los organismos afectados.

Veamos, a continuación, las lógicas que subyacen a cada uno de estos modelos:

El primer modelo revaloriza la Formación Continua, remarcando que la misión de la Universidad es poner en marcha, permanentemente, procesos educadores a lo largo de la carrera del individuo. Se trata de favorecer el retorno a la Universidad de aquellos adultos comprometidos con su propia formación y desarrollo integral, reconociendo y valorando desde la Universidad la formación y conocimientos adquiridos a través de años de experiencia. 
El segundo modelo llamado deslocalizado usa dos lógicas no convergentes. Se trata de la acción de docentes comprometidos con la necesidad de favorecer el acceso a la formación de personas que disponen de una experiencia personal y profesional amplia, y que no encuentran en sus contextos de desarrollo los medios institucionales necesarios que les reconozcan y potencien las competencias adquiridas. Los docentes, sea a título individual o colectivo, son los motores de este segundo modelo.

El tercer modelo se identifica con la lógica de la conformidad. La Administración de la Universidad tiene como preocupación el hacer posible la aplicación de una legislación relativa a la Validación de Competencias, pero no tiene los medios adecuados para imponer un dispositivo que supere su grado de intervención.

\subsection{TRES MODELOS DE VALIDACIÓN DE COMPETENCIAS}

Sin embargo, los modelos a los que nos venimos refiriendo que son desarrollados desde las Universidades, se sitúan como parte integrante de modelos mucho más amplios que hacen referencia a diferentes concepciones de la validación en la Educación Superior. Estos macromodelos son básicamente tres:

\section{A. Modelo Escolar}

Podemos decir que hay un primer modelo que pone el acento en la "PRUEBA" a proporcionar por parte del candidato. Este modelo es particularmente defendido por J. Aubret (1990, 1991, 1992 y 1994). El acceso a la preparación de un diploma no quiere decir dispensar de presentar las pruebas de haber adquirido los conocimientos. Igualmente la obtención, según el Decreto de 1993, supone "la presentación de una prueba fehaciente". Este modelo habla de lo que se llama "memoria profesional"; ésta debe ser el resultado de un trabajo de descripción, de análisis, de formalización, de síntesis de las experiencias personales y profesionales que el candidato quiere validar. Toda la responsabilidad, según este modelo, se centra sobre el candidato; a él le corresponde el comprender las reglas del juego y demostrar que es capaz de producir elementos ajustándose a una prueba de la institución universitaria. Se puede decir que la institución no modifica sus procedimientos y sus criterios, sino que los adapta a una situación particular.

\section{B. Modelo Militante (Portafolios)}

El segundo gran modelo se basa en lo que hemos denominado, metodologías portafolio. Se trata en un proceso de análisis del conjunto de las expe- 
riencias personales, sociales y profesionales que, a través de un proceso de confrontación, organización y síntesis, ayudará al individuo a extraer sus propias conclusiones acerca de las competencias adquiridas, facilitando así la negociación entre las partes, dentro del Proceso de Validación de Competencias. Estas metodologías suelen demandar la presentación de elementos de prueba que demuestren el dominio de las competencias del candidato.

El dossier reunirá, al menos, el Currículum Vitae del individuo, documentación alusiva a la formación del futuro alumno, títulos y diplomas varios, certificaciones de los trabajos realizados así como referencias de los lugares en los que haya desempeñado su labor profesional.

Este modelo vuelve a abogar por potenciar la responsabilidad y autonomía del candidato, aunque no sólo en las fases iniciales del Proceso de Validación sino también a lo largo de las mismas.

\section{Modelo del Ministerio de Educación Nacional}

El tercer modelo que ha sido propuesto por el Ministerio de Educación Nacional en Enseñanza Superior es el resultado de los trabajos de los diferentes grupos de reflexión, a los que aludíamos en apartados anteriores al referirnos a la necesidad de coordinación entre los sectores productivos y académicos, siendo éstos los encargados de poner a punto una metodología de validación basada en los decretos 85/906 y 93/489. Esta metodología, en ciertos aspectos, está próxima al proceso del portafolios, aunque simplifica el procedimiento y la producción del dossier. Proponen dos tipos de dossier:

- El primer dossier aplica el Decreto 85/906, proponiendo una presentación resumida acerca de la carrera personal y profesional del candidato.

- El segundo dossier aplica el Decreto 93/489, incitando al candidato a analizar y plasmar, de manera precisa, la actividad profesional que va a servir de soporte al proyecto de validación. El proceso preconiza una relación viva entre el candidato y un asesor de formación y, eventualmente, docentes para así obtener un desarrollo completo del dossier de validación, identificando las competencias consideradas como previas al inicio del Proceso de Validación.

Por otro lado, destacaremos que los criterios de evaluación son diferentes en función del tipo de dossier empleado. Veámoslo:

a) En el marco del Decreto 85/906, se presenta una apreciación global sobre el itinerario personal y profesional del candidato, sobre su recorrido de formación inicial y continua. Se va a intentar identificar los esquemas de acción, los procesos de resolución de problemas puestos 
en marcha por el sujeto, los procesos de reflexión puestos en juego en el conjunto de actividades y el nivel de responsabilidad de sus actos. Se parte de la hipótesis de que los elementos mencionados anteriormente son indicadores veraces de las competencias y potencialidades previas requeridas para optar al proceso de obtención de una determinada cualificación.

b) En cuanto al dossier basado en el Decreto 93/489, el proceso de elaboración reposa sobre los mismos principios que el anterior, aunque con una diferencia significativa, se tratará de atribuir unidades de valor sobre la base de unas competencias ya adquiridas. El trabajo de análisis reposará, por tanto, sobre la descripción razonada y profunda de la actividad profesional que va a servir de base a la petición de validación. El objetivo que se persigue es determinar la zona de desarrollo próximo en la que se sitúa el demandante de una determinada certificación en relación con las competencias que componen dicha certificación. Pero lo que diferencia este proceso de otros, incluso las experiencias anglosajonas, es que éste responsabiliza a la Institución de la elaboración de informes adecuados que permitan la toma de decisiones adecuadas. La Universidad garantiza al candidato la existencia de un dispositivo que le va a permitir determinar con toda claridad y eficacia sus competencias previas así como la presentación de los elementos más contundentes que lo demuestren.

Con esto he terminado lo que podríamos llamar la presentación global de los modelos de enseñanza en Francia y modelos de validación en general.

Vamos a estudiar algunos modelos más próximos de aplicar entre nosotros.

\section{MÉTODO Y PROCESO DE VALIDACIÓN EN LA ENSEÑANZA SUPERIOR EN FRANCIA. COLEGIOS COOPERATIVOS}

La metodología y proceso de validación presentados a continuación aluden a una nueva forma de validar las diversas competencias aglutinadas en el marco de la Enseñanza Superior francesa. Estos nuevos métodos y procesos de validación están siendo desarrollados, de manera destacada y exclusiva, por los llamados Colegios Cooperativos, los cuales han servido de base para la realización de la investigación sobre la cual se fundamentan los datos aportados en el presente apartado, destacando las experiencias realizadas en los siguientes centros educativos: 
- Colegio Cooperativo en Bretaña: Universidad Rennes.

- Colegio Cooperativo en Provence: Universidad Aix en Provence.

- Colegio Cooperativo de París: Montrouge.

- Colegio Cooperativo Rhône Alpes: Lyon.

- Universidad de Maine: Le Mans.

- Conservation National de Artes y Oficios: Chans París.

- Universidad de Toulouse.

- Universidad de Francia: Condado Besançon.

- Sorbona Nueva. París III.

A lo largo de este apartado trataremos de describir:

- El método y el proceso de validación seguido en un programa a tal fin, de tres años de duración.

- La metodología y el proyecto que subyacen al programa de validación.

- La metodología experimentada, la cual se centrará en dos niveles: El autobiográfico y el de realización del proyecto de validación.

Finalmente, destacar que el modelo aludido se sitúa en la línea descrita en el capítulo anterior correspondiente a las Metodologías Portafolio, siendo el autor intelectual del modelo M. Desroche a partir de la publicación de sus dos obras clave:

Iniciación a las Ciencias Sociales (1984) y Entreprende d'apprendre (1991).

\subsection{DIPLOMA DE ALTOS ESTUDIOS DE PRÁCTICAS SOCIALES (DHEPS): EL DIPLOMA DE ALTOS ESTUDIOS DE PRÁCTICAS SOCIALES COMO FORMACIÓN PARA LA INVESTIGACIÓN/ACCIÓN}

\section{A. El éxito de la investigación/acción}

Desde 1985 a 1995 el número de demandas para obtener un diploma que validase las competencias obtenidas en el campo de los servicios sociales, por diferentes sectores profesionales, basándose este proceso en un modelo de 
certificación de competencias profesionales, ha ido aumentando enormemente, desembocando todo ello en la constitución y consolidación del conocido como Diploma de Altos Estudios de Prácticas Sociales, gracias, a su vez, al éxito de las diferentes experiencias llevadas a cabo a través de un modelo de investigación/acción.

\section{B. El diploma de altos estudios de prácticas sociales}

Este diploma corresponde a la obtención de un nivel académico de segundo ciclo universitario. El diploma es entregado por varias universidades francesas, las cuales se agrupan en la llamada Red de Altos Estudios en Prácticas Sociales, más conocidas por las siglas G.I.S, avaladas desde el Ministerio de Educación.

\section{c. Destinatarios}

La formación implementada por este Diploma representa 350 horas por año durante tres años. Reagrupa a una población muy diversa, si tenemos en cuenta las profesiones representadas y los campos sobre los cuales van a construirse los objetos de investigación, aunque los candidatos pertenecen, todos ellos, al sector terciario. Suelen ser profesionales del trabajo social (enfermeras, puericultoras, etc.), agentes de desarrollo local y formadores, tanto de organismos privados como públicos, tales como directores de Casas de la Juventud y de Servicios Municipales, responsables de servicios asociativos; animadores de centros socioculturales, responsables sindicales, directores de servicios de atención a personas con minusvalías o que fomentan la reinserción de jóvenes en riesgo de exclusión social, trabajadores sociales responsables de programas de desarrollo para los países del tercer mundo; asesores de ANPE o ASEDIC y responsables de servicios de mutualidades de diversas cooperativas.

\section{Fase inicial}

¿Qué es lo que unifica a estas personas que proceden de ámbitos tan diversos? La respuesta está en la homogeneidad de sus motivaciones, en relación con su ámbito laboral. Cada uno de los aspirantes piensa que este diploma le permitirá pasar de una práctica intuitiva a una práctica razonada, reflexionada, científicamente consolidada.

\section{E. La investigación/acción}

La investigación/acción es un proceso metodológico basado en la reflexión sobre la propia práctica de los actores que forman parte del proceso investi- 
gador al objeto de provocar un proceso de mejora constante de la práctica mencionada. Esta metodología será la predominante en las primeras fases del desarrollo del proceso de validación de las competencias profesionales a las que nos venimos refiriendo.

\section{F. Dialéctica, presencia y distancia}

La utilización del modelo de investigación/acción implica un fuerte proceso de abstracción por parte de los participantes. Con ello, se trata de que se abstraigan de su propia práctica para poder juzgarla en su globalidad. Toda la calidad del proceso investigador va a determinarse en esta dialéctica entre la implicación y la distancia del investigador/actor.

A continuación, pasamos a describir las diferentes fases y elementos del proyecto objeto del presente apartado.

\subsection{PRIMER AÑO: PLANTEAMIENTO Y REDACCIÓN DEL PROYECTO}

\section{Historia de vida}

Las sesiones de formación, dos días por mes, comienzan con el análisis de las prácticas de los candidatos y de las preguntas que se formulan como punto de partida a su reflexión. Ello constituye un primer ensayo de lo que llegará a ser el proceso de desarrollo del proyecto de investigación/acción. Ello Será regularmente evaluado, comentado, reorientado, tutorizado permanentemente, por uno de los profesores integrados en equipo de docentes del DEPS (Diploma de Altos Estudios de Prácticas Sociales).

\section{Iniciación}

Posteriormente se lleva a cabo una introducción a los conceptos más significativos de las Ciencias Sociales, por una parte, y a los métodos de investigación y a sus exigencias, por otra, estableciendo cada candidato conexión de todo ello con su práctica profesional, de forma escrita.

\section{Definición}

Los participantes determinarán su propio campo de la investigación, su situación respecto del proceso de investigación, el objeto de la investigación, sus hipótesis de trabajo y los métodos previstos para validar las hipótesis. 


\section{4..$^{\circ}$ Redacción}

Elaboración de un proyecto de investigación/acción, con una extensión aproximada de 30 páginas, en el que se desarrollen los cinco puntos que acabamos de enumerar:

- Campo de la investigación.

- Situación del investigador-actor.

- Objeto de la investigación.

- Hipótesis de trabajo.

- Métodos de validación de hipótesis.

\section{Formación complementaria}

Este aprendizaje conceptual y metodológico se complementa con:

- Formación en los métodos de recogida de información (cuestionarios de diverso tipo, etc.).

- Formación en cuanto a las diversas exigencias del discurso científico.

- Iniciación a los conceptos y a las técnicas del análisis del discurso científico.

- Formación sobre el lenguaje como fuente de información de la realidad social.

\subsection{SEGUNDO AÑO: REALIZACIÓN DEL PORTAFOLIOS O PROYECTO DE INVESTIGACIÓN}

\section{Trabajo en grupo}

La pedagogía de la puesta en marcha en las diferentes sesiones se basará en exposiciones orales por parte del docente, debates en torno a los asuntos expuestos, trabajo en pequeño o gran grupo, en función de la naturaleza de la actividad presentada, y puesta en común de las reflexiones desarrolladas por el alumnado, tanto a nivel individual como grupal. El docente será aquí un mediador entre los conocimientos a adquirir y el propio alumno, ayudándole a éste a recorrer la senda de su desarrollo cognitivo.

\section{Diario de campo}

Además de esta metodología de carácter interactiva, el aspirante irá construyendo, a partir del trabajo realizado en cada sesión, su diario de campo. 
Éste contendrá la evolución de sus reflexiones fruto de su interacción con las del resto de sus compañeros y con las suyas propias, manifestadas en forma de conocimientos previos. Deberá reflejar la forma en la que sus estructuras cognitivas, pertenecientes al área de conocimiento trabajada, van evolucionando, desarrollando y madurando competencias diversas, todo ello bajo la guía de su tutor personal que irá evaluando este proceso. Ello derivará en la presentación de un proyecto de investigación-acción de carácter científico, que será sometido al consejo de un DEPS para la admisión el segundo año de este alumno y la entrega de un certificado de capacidad en investigación.

\subsection{TERCER AÑO: REDACCIÓN FINAL DEL PROYECTO Y DEFENSA DEL MISMO}

\section{Tipos de evaluación}

a. Evaluación formativa: Permitirá la orientación del alumnado, regularmente ejercitada por el equipo permanente del DEPS.

b. Evaluación sumativa: Destinada a medir el grado de conocimientos adquiridos en un momento dado del proceso formativo, como sucedió al finalizar el primer año, que culminó con la entrega de un certificado de capacidad en investigación-acción, y a la conclusión del segundo año, al someter a examen la memoria del trabajo realizado delante de un jurado en el que participará el tutor del alumno correspondiente.

\section{Memorias}

Las memorias a las que nos venimos refiriendo se convierten en una base de datos de conocimientos frecuentemente transferibles al campo científico al que hacen referencia. Es por ello por lo que estas memorias se constituyen en auténticas bases de datos que los DEPS de las catorce Universidades participantes gestionan, poniéndolas a la disposición del alumnado de estas Universidades para futuros procesos investigadores en los diferentes campos científicos a los que aluden, contribuyendo además no sólo al avance científico en estos campos, sino al perfeccionamiento de la propia metodología de investigación/acción, imprescindible para el estudio de fenómenos de interacción social.

\section{MÉTODOLOGÍA Y PROCESO DE VALIDACIÓN. MODELO ESCOLAR}

El proceso y la metodología que aquí vamos a presentar corresponde a un modelo descrito anteriormente y que hemos llamado de carácter "más escolar". Este proceso se basa eminentemente en la PRUEBA y en una prueba mucho más 
de carácter intelectual, mucho más escolar. Podemos decir que este proceso prácticamente sigue una metodología común en las escuelas, en los centros escolares. Vamos a abordar el tema en dos etapas: Una primera etapa describiendo la metodología del portafolio como proceso y como contexto en el cual se va a trabajar el tema, y otra segunda parte utilizando herramientas más precisas y más apropiadas. Nos basaremos fundamentalmente en dos autores:

- En la primera parte utilizaremos la metodología canadiense del portafolio y en concreto los trabajos Ginette Robin (1992).

- En la segunda parte seguiremos la tradición más francesa basada en los trabajos de Jacques Aubret $(1990,1992)$.

\subsection{CARÁCTER GENERAL DEL PORTAFOLIO}

El método Portafolio en nuestro proceso de validación implica, a su vez, el desarrollo de un proceso de reconocimiento y la obtención de un producto documental.

\section{A. El portafolio como proceso}

El Portafolio como proceso posee cinco etapas a seguir por el aspirante bajo la dirección de un tutor personal.

\section{Preparación}

Definición de objetivos por parte del alumno y determinación del proceso a seguir para la obtención de los mismos.

\section{2. ${ }^{\circ}$ Revisión histórica de su vida}

Elaboración del Currículum Vitae del aspirante en el que se describa la carrera profesional y personal seguida por el individuo.

\section{Inventario de Aprendizajes}

Identificación de los aprendizajes adquiridos relacionados con la certificación a la que se opta y autoevaluación de los mismos.

\section{4. ${ }^{\circ}$ Traducción de los Aprendizajes}

Determinación del grado de adecuación y correspondencia de las competencias adquiridas en relación con la certificación solicitada y bajo los criterios de la Institución que valida la mencionada certificación. 


\section{Demostración}

El individuo ha de ser consciente de que las competencias que vaya adquiriendo deberán ser puestas en práctica no sólo ante la Institución que le evalúa sino también ante la Empresa a la que pertenece o la que le pretende emplear, de forma permanente a lo largo de su vida laboral.

\section{B. El portafolio como producto}

El Portafolio es en sí mismo un dossier personal, avalado por la documentación correspondiente a tal efecto dentro de un proceso de sistematización, constituido para determinar y validar las competencias de cada aspirante por parte de la Institución que lo otorga y/o de la Empresa que lo demanda. Dicho documento contendrá los siguientes elementos:

- Exposición de motivos por los que se solicita y requiere la certificación de las competencias objeto del proceso de validación.

- Currículum Vitae respecto de la carrera personal y profesional del aspirante.

- Síntesis cronológica de experiencias y aprendizajes que dieron lugar a la adquisición de las competencias previas necesarias para acceder al proceso de validación.

- Cuadro de presentación de competencias adquiridas a lo largo del proceso de validación.

- Documentación y pruebas acreditativas de la obtención de la certificación de las competencias profesionales demandadas por la persona participante.

Podemos observar, en este caso, que el proceso metodológico y los apartados que lo componen están muy próximos al desarrollado en el contexto escolar de manera habitual.

\section{VALIDACIÓN DE COMPETENCIAS Y CERTIFICACIÓN DE CUALIFICACIONES PROFESIONALES EN LAS EMPRESAS FRANCESAS}

\subsection{ORIGEN Y FINALIDAD DE VALIDACIÓN DE LAS CERTIFICACIONES DE CUALIFICACIONES}

En el caso de la comisión paritaria de la metalurgia, la puesta en marcha tiene estas fechas: 
JULIO DEL 86. Aparece la ordenanza n. ${ }^{\circ} 86-836$ que posibilita hacer contratos de cualificación en la empresa.

12 JUNIO DEL 87. Acuerdo nacional sobre los problemas generales del empleo en la Unión de Industrias Minerometalúrgicas y los sindicatos mayoritarios. Se pone en marcha una comisión paritaria del empleo.

4 ENERO DEL 88. Se constituye un grupo técnico paritario, fruto de la comisión nacional paritaria de la metalurgia, encargado de elaborar reglas de validación de cualificaciones.

SEPTIEMBRE DEL 88. Se aprueba la comisión paritaria nacional del empleo de la metalúrgica; se aprueban por la comisión las reglas definitivas del grupo técnico paritario.

Primeras decisiones de validación tomadas por el grupo técnico paritario

ENERO DEL 90. Conclusión de un acuerdo nacional. En los términos de este acuerdo, la comisión paritaria nacional de la metalurgia, está encargada de la clasificación de cualificaciones validadas en vistas de aplicación

OCTUBRE DEL 90. Aprobación por la comisión nacional paritaria del empleo y de la metalurgia de las reglas definidas por el grupo técnico paritario.

JULIO DEL 92. Se concluye un nuevo acuerdo nacional en el sector del metal

COMENTARIO MARZO DEL 93. Se definen las normas del empleo y de las certificaciones.

\subsection{SISTEMA DE VALIDACIÓN PARITARIA DE LAS CUALIFICACIONES}

Después, como he dicho, y a partir de estas fechas, se ha puesto en marcha lo que se llama un "sistema paritario de validación de las cualificaciones" con varias condiciones. Las dificultades del contrato de cualificación encontradas en el proceso llevaban a interrogarse sobre el puesto ocupado por las profesiones en las cualificaciones, así como las relaciones que deben mantener; la medida del contrato de cualificación con la del contrato de aprendizaje; sin omitir el necesario tema de los medios a movilizar para salir del marco de una oferta de formación preestablecida y por citar más formalmente a las empresas a centrarse en las actas de formación de necesidades. Por ello se puso en marcha el dispositivo de validación paritaria de las cualificaciones, lo cual suponía una ruptura y una originalidad: 
- La cualificación comporta la definición de un objetivo profesional; sus componentes debían estar expresados en términos de "ser capaz de".

- La cualificación comporta la definición de un instrumento de medida que puede ser un control final, una memoria con defensa o cualquier otro dispositivo.

- Su identificación y su definición son responsabilidad de la profesión.

- Una combinación estrecha a escala nacional y territorial constituye la última característica de este dispositivo de validación paritaria de las cualificaciones; por lo tanto la identificación y definición de necesidades son efectuadas bajo la tutela de las cámaras sindicales territoriales.

- Las etapas para poner en marcha la profesión suponen estos pasos: Identificación de necesidades; definición de objetivos profesionales y pruebas de cualificación correspondientes; validación de objetivos profesionales y de pruebas de cualificación; paso de las pruebas de cualificación; deliberación de jurado; atribución de certificados de cualificación paritaria. Todo ello tenía como finalidades claras:

1. Permitir el desarrollo del control de cualificación paralelamente a los contratos de aprendizaje, cuya progresión queda como una de las prioridades importantes.

2. Invitar a las empresas a definir sus necesidades y los medios de satisfacerlas.

3. Poner en marcha los dispositivos de formación de la profesión para poner en marcha formaciones diferenciadas

4. Dotar a la profesión de un sistema de certificación que le sea propia y de unos centros de validación o de certificación de las competencias adquiridas.

5. Impulsar un nuevo modelo de relaciones entre los niveles nacionales y territoriales.

6. Permitir a las comisiones paritarias de empleo una implicación más fuerte en los temas de formación.

Se es consciente de haber realizado una presentación muy resumida de un proyecto amplio que exigiría mayor desarrollo.

Sin embargo, se tiene la esperanza de que iniciativas que van surgiendo en España puedan hacer un trabajo más amplio en el campo de la Validación de Competencias Profesionales. 


\section{BIBLIOGRAFÍA}

AUBRET. (1992): Reconnaissance et validation des acquis: le portefeuille de compétences. Paris. Universidad Paris VIII.

AUBRET, J; AUBRET, F. y DAMIANI, C. (1990): Les Bilans personnels et professionnels. Guide métholologique. Issy - Les Moulineaux Editions EAP,

AUBRET, J. GILBERT P. (1991): Reconnaissance et validation des acquis. Paris.PUF.

AUBRET, J. MEYER N. (1994): La reconnaissance des acquis personnels et professionnels et l'enseignement supérieur les enjeux, Pratiques et analyses de formation. Paris. Université de Paris VIII
CEDEFOP (1992) : Correspondencia de cualificaciones en Europa. Berlín. Cedefop.

CHARRAUD A.M., (1994): A propos d'un glossaire sur la validation des acquis, document de travail, Arcueil. Francia.

DESROCHE, HENRI (1984): Iniciación a las Ciencias Sociales. Prólogo de R. Barthes. Barcelona, Hogar del Libro. Colección Nova Terra

DESROCHE, HENRI (1991): Entreprendre d'appendre. Paris, Les Editions Ouvrières

ROBIN, GINNETTE (1992): Guide en Reconnaissance des Acquis. Québec Vermette

\section{RESUMEN}

El articulo ofrece un resumen de la filosofía y marco que el tema de "Validación de Competencias Profesionales en Francia".

Se aborda el tema general de homologación, se ofrecen modelos de realización según las distintas instancias y se ofrece una bibliografía amplia.

Palabras clave: Validación, Competencias Profesionales, Homologación, Certificación.

\section{ABSTRACT}

The article offers a summary of the philosophy and mark that the topic of "Validation of Professional Competitions in France."

The general topic of approval is approached, realization models offer according to the different instances and he/she offers a wide bibliography. 


\section{ANEXOS}

\section{TABLA I}

\section{Los procesos de elaboración de diplomas, títulos y CQP}

\begin{tabular}{|c|c|c|c|c|c|c|}
\hline $\begin{array}{c}\text { Lugar de } \\
\text { concep- } \\
\text { ción del } \\
\text { Titu- } \\
\text { lo }\end{array}$ & $\begin{array}{c}\text { Ministerio } \\
\text { de Educación } \\
\text { Nacional }\end{array}$ & $\begin{array}{c}\text { Ministerio } \\
\text { de Agricultura }\end{array}$ & $\begin{array}{l}\text { Ministerio } \\
\text { de Trabajo }\end{array}$ & $\begin{array}{c}\text { Ministerios } \\
\text { de: Comercio } \\
\text { y Artesania, } \\
\text { Salud, } \\
\text { Juventudy } \\
\text { Deportes, }\end{array}$ & $\begin{array}{c}\text { Organismos } \\
\text { de formación } \\
\text { (entre los que } \\
\text { están las } \\
\text { universidades) } \\
\text { o empresas } \\
\text { Defensa, Mar }\end{array}$ & $\begin{array}{c}\text { Ramas } \\
\text { profesionales }\end{array}$ \\
\hline $\begin{array}{l}\text { Consulta } \\
\text { técnica }\end{array}$ & $\begin{array}{l}\text { C.P.C. } \\
\text { (Comisión } \\
\text { profesional } \\
\text { consultiva) }\end{array}$ & $\begin{array}{l}\text { C.P.C. } \\
\text { (Comisión } \\
\text { profesional } \\
\text { consultiva) }\end{array}$ & $\begin{array}{l}\text { C.P.C. } \\
\text { (Comisión } \\
\text { profesional } \\
\text { consultiva) }\end{array}$ & $\begin{array}{l}\text { En el seno de } \\
\text { cada } \\
\text { ministerio en } \\
\text { relación con } \\
\text { los } \\
\text { representantes } \\
\text { de la } \\
\text { profesión }\end{array}$ & & \multirow[t]{2}{*}{$\begin{array}{l}\text { En el seno de } \\
\text { comisiones } \\
\text { paritarias del } \\
\text { empleo } \\
\text { cuando estas } \\
\text { existen }\end{array}$} \\
\hline $\begin{array}{l}\text { Consulta } \\
\text { previa de la } \\
\text { validación } \\
\text { oficial }\end{array}$ & & $\begin{array}{l}\text { C.T.H. } \\
\text { (Comisión } \\
\text { técnica de } \\
\text { homologación) } \\
\text { Únicamente } \\
\text { para los títulos } \\
\text { homologados }\end{array}$ & \multicolumn{3}{|c|}{$\begin{array}{l}\text { Comisión técnica de homologación de títulos } \\
\text { y diplomas de enseñanza tecnológica }\end{array}$} & \\
\hline $\begin{array}{l}\text { Decisión } \\
\text { oficial de } \\
\text { certificación }\end{array}$ & $\begin{array}{l}\text { Ministerio de } \\
\text { educación }\end{array}$ & \begin{tabular}{|l|} 
Diplomas. \\
Ministros de \\
educación \\
nacional y de \\
agricultura. \\
Títulos. \\
Ministro de \\
trabajo y de \\
la formación \\
profesional, \\
por delegación \\
del Primer \\
Ministro
\end{tabular} & $\begin{array}{l}\text { Ministro de } \\
\text { trabajo y de } \\
\text { la formación } \\
\text { profesional } \\
\text { por } \\
\text { delegación } \\
\text { del Primer } \\
\text { Ministro }\end{array}$ & $\begin{array}{l}\text { Ministerio de } \\
\text { trabajo y de } \\
\text { la formación } \\
\text { profesional } \\
\text { por } \\
\text { delegación } \\
\text { del Primer } \\
\text { Ministro }\end{array}$ & $\begin{array}{l}\text { Ministerio de } \\
\text { trabajo y de } \\
\text { la formación } \\
\text { profesional } \\
\text { por } \\
\text { delegación } \\
\text { del Primer } \\
\text { Ministro }\end{array}$ & \begin{tabular}{|l|} 
Comisiones \\
paritarias del \\
empleo de la \\
rama \\
profesional. \\
Puede pedir \\
la \\
homologación \\
de los títulos
\end{tabular} \\
\hline $\begin{array}{l}\text { Naturaleza } \\
\text { de la } \\
\text { certificación }\end{array}$ & $\begin{array}{l}\text { Diplomas } \\
\text { nacionales }\end{array}$ & $\begin{array}{l}\text { Títulos } \\
\text { homologados } \\
\text { y diplomas } \\
\text { nacionales }\end{array}$ & $\begin{array}{l}\text { Títulos } \\
\text { homologados }\end{array}$ & $\begin{array}{l}\text { Títulos o } \\
\text { diplomas de } \\
\text { Estados, } \\
\text { homologados } \\
\text { o no }\end{array}$ & $\begin{array}{l}\text { Títulos } \\
\text { homologados }\end{array}$ & $\begin{array}{l}\text { Cualificación } \\
\text { reconocida o } \\
\text { Cualificación } \\
\text { profesional o } \\
\text { título } \\
\text { homologado }\end{array}$ \\
\hline
\end{tabular}

Flente: CEDEFOP PANORAMA (1994b) 


\section{TABLA II}

\section{Siglas, términos técnicos usados en Francia. (Resumen)}

- ASEDIC: Asociación para el Empleo en la Industria y el Comercio.

- APEL: Agencia nacional de empleo de cuadros.

- AFPA: Asociación para la formación profesional de adultos.

- ANPE: Agencia nacional para el empleo.

- BASE: Diploma de aptitudes de animación socioeducativa.

- BEATEP: Diploma de animador técnico de educación.

- BEES: Diploma de Estado de educador deportivo.

- BEP: Diploma de estudios profesionales.

- BT: Diploma de técnico.

- BTS: Diploma de técnico superior.

- CAFAMP: Certificado de aptitudes para la función de médico-psicólogo.

- CAFAD: Certificado de aptitudes para la función de ayuda a domicilio.

- CAFM: Certificado de aptitudes para la función de monitor de educación.

- CEP: Certificado de estudios primarios.

- CEREQ: Centro de estudios y de investigación de la cualificación.

- CFI: Crédito de formación individualizada.

- CHT: Comisión de homologación de títulos y diplomas.

- CIBS: Centro interministerial de balance de competencias.

- CNPE: Comisión nacional paritaria del empleo.

- CPA: Clase preparatoria para la alternancia.

- CPC: Comisiones paritarias consultivas.

- CREPS: Centros regionales de educación física y deportiva.

- DEA: Diploma de Estudios Especializados.

- DEES: Diploma de Estado de educación especializada.

- DEFA: Diploma de Estado relativo a las funciones de animación.

- EPS: Diploma de Altos Estudios en Practicas Sociales

- DESS: Diploma de Estado de servicio social.

- DEUG: Diploma de estudios universitarios generales.

- DEUST: Diploma de estudios universitarios científicos y técnicos.

- DRAF: Dirección regional de la Agricultura y Bosque.

- DRASS: Dirección Regional de la acción sanitaria y social.

- DUT: Diploma universitario tecnológico.

- EFAA: Examen de fin de aprendizaje artesanal.

- ESEU: Examen especial de entrada en la universidad.

- FONGECIF: Fondo de gestión de permisos individualizados de formación.

- INSEE: Instituto nacional de estadística y de estudios económicos.

- MASI: Ministerio de Asuntos Sociales y de la Integración.

- OPACIF: Organismo paritario vinculado al permiso individualizado de formación.

- SES: Sección de educación especial.

- STS: Sección de técnicas superiores.

- UCARE: Unidades capitalizables de adaptación regional o de empleo.

- UIMM: Unión de industrias metalúrgicas y mineras. 